\title{
The role of Lys525 on the head-group anchoring of fatty acids in the highest affinity binding site of albumin
}

\author{
Bruno Rizzuti ${ }^{\mathrm{a}, *}$, Manuela Pantusa ${ }^{\mathrm{b}}$ and Rita Guzzi ${ }^{\mathrm{b}}$ \\ ${ }^{\mathrm{a}}$ LiCryL CNR-INFM and CEMIF.Cal, University of Calabria, 87036 Rende, Italy \\ ${ }^{\mathrm{b}}$ Department of Physics and Unità CNISM, University of Calabria, 87036 Rende, Italy
}

\begin{abstract}
Human serum albumin provides the transport of long-chain fatty acids in the blood through three high-affinity and four low-affinity binding sites. Molecular dynamics simulations have been performed to investigate the anchoring of palmitic acid molecules to the protein. In the site with the highest affinity, Site 5, the key residue Lys525 not only binds the head-group of the palmitate ion by electrostatic interactions with its charged terminal group, but it also accommodates the first portion of the lipid chain by non-electrostatic interactions with the rest of its sidechain. The flexibility of Lys525, and in particular of the dihedral angle $\chi_{3}$, is suggested to account for a number of spectroscopic properties observed in correspondence with the entrance of the hydrophobic pocket constituting Site 5.
\end{abstract}

Keywords: Human serum albumin, palmitic acid, lysine, dihedral angle, molecular dynamics

\section{Introduction}

Human serum albumin is the most abundant protein in the blood, providing the transport of fatty acids (FAs) and a variety of other compounds, including metabolites, drugs and ions. Long-chain FAs can attach at seven binding sites [1]: four with low affinity (Site 1,3,6 and 7) and three with high affinity (Site 2, 4 and 5) [2,3]. In particular, Site 5 has the highest value of binding free energy [2]. The FA molecules are attached non-covalently to the protein by a combination of hydrogen bonds and electrostatic interactions that anchor the carboxylate head-group, and of hydrophobic and steric interactions that confine the methylene chain [4].

In all of the three high-affinity binding sites a hydrogen bond is formed [1,4] between the headgroup of the FA and the sidechain of a Tyr residue (Tyr401 for Site 5). For both Site 2 and 4 additional restraints are provided by another hydrogen bond formed with a Ser residue and by electrostatic interaction with an Arg residue. In contrast, the FA head-group in Site 5 is additionally restrained solely by the Coulombic interaction with Lys525. Thus, from an electrostatic point of view, Lys525 is believed to play a fundamental role in determining the characteristics of the highest-affinity binding site [4]. Nonelectrostatic interactions give also an important contribution in attaching FAs to albumin. In fact, the three high-affinity binding sites consist in long and narrow hydrophobic pockets, whereas low-affinity

\footnotetext{
* Corresponding author: Bruno Rizzuti, LiCryL CNR-INFM and CEMIF.Cal, University of Calabria, 87036 Rende, Italy. Tel.: +390984 49 6078; Fax: +390984 49 4401; E-mail: bruno.rizzuti@cnr.it.
} 
sites are shorter and wider [4]. However, local effects due to hydrophobic interactions are more difficult to estimate in the experiment.

In this work, molecular dynamics simulations have been employed to investigate the effect of the protein dynamics to the anchoring of the head-group of palmitic acid in the highest-affinity binding site of albumin. The results elucidate the details of how Lys 525 contributes to secure the FA molecule in the binding pocket by both electrostatic and non-electrostatic interactions.

\section{Methods}

Molecular dynamics was performed by using the GROMACS package [5] with the GROMOS 53a6 force field [6]. The palmitic acid molecule was built by assembling the carboxylate moiety of Glu/Asp for the head-group, methylene groups of Glu all along the FA chain, and the methyl moiety of Ala for the tail. The starting configuration was modeled on the basis of the crystallographic structure of the palmitateloaded albumin [4], 1E7H entry in the Protein Data Bank, reconstructing the position of missing atoms with the help of VMD software [7]. The protonation state of ionizable residues was adapted to mimic neutral $\mathrm{pH}$ and counterions were added to neutralize the system. The protein was solvated in a rhombic dodecahedron box by adding a hydration shell with a minimum thickness of $0.8 \mathrm{~nm}$, using the SPC water model. Periodic boundary conditions were applied and the NPT ensemble was adopted, by using a Berendsen thermostat and barostat [8] with reference values of $300 \mathrm{~K}$ and $10^{5} \mathrm{~Pa}$ and relaxation times of 0.1 and $1 \mathrm{ps}$, respectively. For non-bonded interaction, a twin-range cutoff of 0.8 and $1.4 \mathrm{~nm}$ was employed, plus a Poisson-Boltzmann reaction field with $\varepsilon_{r}=78$. Covalent bonds were constrained [5] by using the LINCS and SETTLE algorithms, which allowed a time step of 2 fs for integrating the equations of motion. After initial energy minimization, a simulated annealing was performed starting at $250 \mathrm{~K}$, followed by production runs of $5 \mathrm{~ns}$. Two simulations were carried out, with palmitic acid molecules either absent or present in Site 2, 4 and 5.

\section{Results and discussion}

Albumin maintains its characteristic heart-shaped tertiary structure and almost (70\%) all-alpha secondary structure in the simulations. After $2 \mathrm{~ns}$ of equilibration, the root mean square deviation of the $\mathrm{C}^{\alpha}$ atoms is $0.48 \pm 0.04$ and $0.47 \pm 0.02 \mathrm{~nm}$ for the loaded and unloaded protein, respectively. The radius of gyration is, respectively, $2.82 \pm 0.03$ and $2.78 \pm 0.03 \mathrm{~nm}$. Both protein ensembles cluster around a single structure, as determined with the Jarvis-Patrick method [9]. Electrostatics plays a fundamental role in the stabilization of FAs into the hydrophobic pockets of albumin. Hydrogen bonds and salt bridges between protein residues and the carboxylate head-group of the lipids are preserved in each of the highaffinity binding sites in the simulation. In Site 5, the FA molecule fluctuates around its starting position in an almost-extended conformation within the protein subdomain III- $b$, with both the head-group and the tail totally exposed to the solvent. The atomic distance between the $\mathrm{O}^{\eta}$ atom of Tyr401 and the closest carboxylate oxygen is $0.27 \pm 0.02 \mathrm{~nm}$, typical of a donor-acceptor hydrogen bond. The distance between the protonated $\mathrm{N}^{\zeta}$ atom of Lys525 and the closest carboxylate oxygen is $0.30 \pm 0.04 \mathrm{~nm}$. While the phenyl ring of Tyr401 is never in contact with the FA molecule, the four methylene groups in the sidechain of Lys525 interact closely with the corresponding groups in the first portion of the lipid chain through non-electrostatic interactions. 


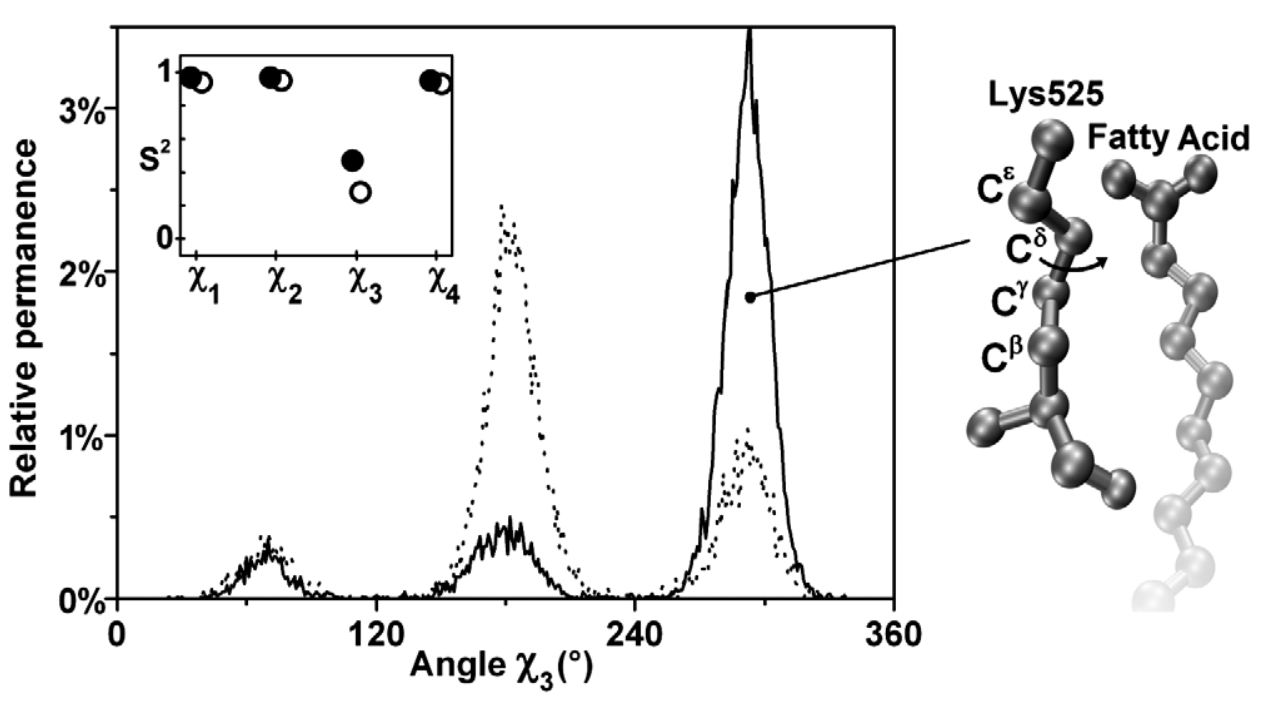

Fig. 1. Relative permanence (ratio between time spent in a given conformation and total simulation time) of the dihedral angle $\chi_{3}$ of Lys525, in the presence (solid line) and absence (dotted line) of palmitic acid, as a function of its value. Lys525 and the fatty acid molecule (with shaded methylene chain) interacting in Site 5 are also shown on the right (only non-H atoms). Inset: Order parameter $S^{2}$ for the four dihedral angles $\chi_{1}, \chi_{2}, \chi_{3}$ and $\chi_{4}$ in the sidechain of Lys525, in the presence (solid circle) and absence (open circle) of palmitic acid.

Figure 1 shows the distribution of values for the dihedral angle $\chi_{3}$ of Lys525 both in the presence (solid line) and absence (dotted line) of palmitic acid. This angle is identified by the position of atoms $\mathrm{C}^{\beta}-\mathrm{C}^{\gamma}-\mathrm{C}^{\delta}-\mathrm{C}^{\varepsilon}$, thus the curves denote rotations around the $\mathrm{C}^{\gamma} \mathrm{H}_{2}-\mathrm{C}^{\delta} \mathrm{H}_{2}$ bond. Minima in the distribution indicate eclipsed configurations and are at $0^{\circ}, 120^{\circ}$ and $240^{\circ}$ (or $-120^{\circ}$, depending on the convention adopted). Maxima correspond to the 3 stable rotamers: gauche ${ }^{+}\left(\chi_{3}=60^{\circ}\right)$, trans $\left(\chi_{3}=180^{\circ}\right)$ and gauche $^{-}\left(\chi_{3}=300^{\circ}\right.$, or $\left.-60^{\circ}\right)$. In both simulations the two gauche isomers deviate from the ideal values, mainly because of the highly-unfavorable cis conformation of the four $\mathrm{C}$ atoms (corresponding to $\chi_{3}=0^{\circ}$ ). The gauche ${ }^{+}$rotamer is the less frequent one, and equally populated with or without the FA. In the absence of the lipid the favored value is $\chi_{3}=180^{\circ}$, corresponding to a planar configuration of the four $\mathrm{C}$ atoms. In contrast, in the presence of the lipid the same rotamer is marginally populated, and the gauche $e^{-}$configuration is distinctively dominant.

As shown in the inset of Fig. 1, the order parameter $S^{2}[10]$ for the $\chi_{3}$ angle is higher in the presence of the FA, confirming that in this case the Lys sidechain is less dynamic. Moreover, rotations around $\chi_{3}$ correspond to the only significant movement of the sidechain of Lys525, because the other relevant dihedral angles possess $S^{2}$ values close to 1 (perfectly rigid). The dihedral angle $\chi_{1}$ is about $70^{\circ}$ both in the presence and absence of the lipid molecule; since this angle is identified by the position of atoms $\mathrm{N}-\mathrm{C}^{\alpha}-\mathrm{C}^{\beta}-\mathrm{C}^{\gamma}$, this indicates that atoms $\mathrm{C}-\mathrm{C}^{\alpha}-\mathrm{C}^{\beta}-\mathrm{C}^{\gamma}$ are always in a trans conformation. The dihedral angle $\chi_{2}$ and $\chi_{4}$ are also found only in a trans conformation. Thus, Lys525 has a clear tendency to possess an extended sidechain in the absence of the FA and to accommodate the lipid molecule, when present, by rotating the sidechain around the $\mathrm{C}^{\gamma} \mathrm{H}_{2}-\mathrm{C}^{\delta} \mathrm{H}_{2}$ bond (see molecular model in Fig. 1).

Residues with long sidechains [11,12] or aromatic rings [13-15] are not uncommon as gatekeepers at the entrance of protein cavities. As an example of the former, Ile99/Leu104/Ile107 hold a pyrrole ring in the heme pocket of myoglobin and participate in the movement required to accommodate carbon monoxyde in its binding site [11]. Instances of the latter include Trp352/Phe356 sealing FK506 into the 
hydrophobic binding site of calcineurin [13], and Trp286/Trp432 regulating the entrance in the substrate binding pocket of $\beta$-D-glucan-glucohydrolase [14]. For albumin, it is known from crystallography that Tyr138 and 161 stack together in the middle of Site 1 to occlude the binding pocket in the absence of a lipid molecule [1], whereas in the presence of the palmitic acid they rotate to hold the ligand [4]. A small steric clamp is evidenced in the simulations at the entrance of Site 5, due to the intrinsic dynamics of the Lys residue. This effect cannot be observed in the X-ray structure, because in the crystal its sidechain is stuck in a gauche $e^{+}$conformation when the protein is complexed with palmitic acid [4].

From an experimental point of view, it is difficult to separate the hydrophobic contribution on FA anchoring in Site 5 due to the four methylene groups in the sidechain of Lys525 from the electrostatic effect due to its terminal protonated amine. As an example, reduced affinity in attaching ${ }^{13} \mathrm{C}$-carboxyllabeled palmitate is detected by X-ray and NMR spectroscopy when Lys525 is mutated into Ala [2]. On the basis of our simulation results, the reduced affinity could be related not only to the elimination of the net charge, but also to suppression of important hydrophobic interactions at the entrance of the protein pocket. On the other hand, non-electrostatic interactions alone are probably insufficient to bind a FA molecule in Site 5, as demonstrated by the fact that no indication of inversion of the ligand within this hydrophobic channel is found in the crystallographic structure [4].

The close interactions observed between the initial portion of the palmitic acid molecule and the sidechain of Lys 525 could explain the higher local heterogeneity observed in X-ray spectroscopy when albumin is complexed with different FAs in this pocket, as compared with the other two high-affinity binding sites. In fact, by superimposing the crystallographic protein structures $[1,4]$ obtained when albumin is loaded with FAs ranging from capric (C10:0) to stearic acid (C18:0), the ensemble obtained at Site 5 is far less uniform as regards the initial portion of the FA anchoring. The simulation results clearly indicate that this should be a consequence of the dynamics of the Lys525 residue.

Electrostatics and steric complementarity of the groove in the protein surface, in correspondence with Site 5, could play an additional role in the covalent and non-covalent binding of various biological molecules. Modifications specifically involving Lys525 include non-enzymatic glycosylation [16] and reaction with a physiological concentration of malondialdehyde [17], the most abundant aldehyde resulting from lipid peroxidation, which forms an N-propenal-lysine adduct. The dynamics of Lys 525 sidechain could be important to determine a selective chemical alteration of this residue, together with the presence of a positively-charged environment [17]. In turn, chemical modifications of Lys525 can affect the ability of albumin to bind FAs. For example, fluorescence data indicate that glycosilated albumin significantly reduces the affinity for cis-parinaric acid [16].

In conclusion, the dynamics of the sidechain of Lys525 exhibits a distinctive rotation around the dihedral angle $\chi_{3}$ in the presence of a lipid molecule in Site 5. Although mainly driven by the Coulombic interactions of the terminal protonated amine group, the dynamics of Lys525 plays an important role also in non-electrostatic interactions and could contribute in determining the key features of the highestaffinity binding site of albumin.

\section{References}

[1] S. Curry, H. Mandelkow, P. Brick and N. Franks, Nature Struct. Biol. 5 (1998), 827-835.

[2] J.R. Simard, P.A. Zunszain, C.-E. Ha, J.S. Yang, N.V. Bhagavan, I. Petitpas, S. Curry and J.A. Hamilton, Proc. Natl. Acad. Sci. USA 102 (2005), 17958-17963.

[3] J.R. Simard, P.A. Zunszain, J.A. Hamilton and S. Curry, J. Mol. Biol. 361 (2006), 336-351.

[4] A.A. Bhattacharya, T. Grune and S. Curry, J. Mol. Biol. 303 (2000), 721-732. 
[5] D. van der Spoel, E. Lindahl, B. Hess, G. Groenhof, A.E. Mark and H.J.C. Berendsen, J. Comp. Chem. 26 (2005), 17011718.

[6] C. Oostenbrink, A. Villa, A.E. Mark and W.F. van Gunsteren, J. Comput. Chem. 25 (2004), 1656-1676.

[7] W. Humphrey, A. Dalke and K. Schulten, J. Molec. Graphics 14 (1996), 33-38.

[8] H.J.C. Berendsen, J.P.M. Postma, A. Di Nola and J.R. Haak, J. Chem. Phys. 81 (1984), 3684-3690.

[9] R.A. Jarvis and E.A. Patrick, IEEE Trans. Comp. 22 (1973), 1025-1034.

[10] D.C. Chatfield, A. Szabo and B.R. Brooks, J. Am. Chem. Soc. 120 (1998), 5301-5311.

[11] G.S. Kachalova, A.N. Popov and H.D. Bartunik, Science 284 (1999), 473-476.

[12] M.A. Scorciapino, A. Robertazzi, M. Casu, P. Ruggerone and M. Ceccarelli, J. Am. Chem. Soc. 131 (2009), 11825-11832.

[13] J.P. Griffith, J.L. Kim, E.E. Kim, M.D. Sintchak, J.A. Thompson, M.J. Fitzgibbon, M.A. Fleming, P.R. Caron, K. Hsiao and M.A. Navia, Cell 82 (1995), 507-522.

[14] J.N. Varghese, M. Hrmova and G.B. Fincher, Structure 7 (1999), 179-190.

[15] M. Hrmova, R. De Gori, B.J. Smith, J.K. Fairweather, H. Driguez, J.N. Varghese and G.B. Fincher, Plant Cell 14 (2002), $1033-1052$.

[16] N. Shaklai, R.L. Garlick and H.F. Bunn, J. Biol. Chem. 259 (1984), 3812-3817.

[17] T. Ishii, S. Ito, S. Kumazawa, T. Sakurai, S. Yamaguchi, T. Mori, T. Nakayama and K. Uchida, Biochem. Biophys. Res. Commun. 371 (2008), 28-32. 


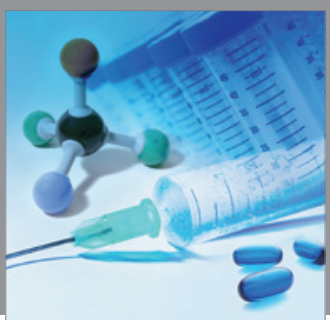

International Journal of

Medicinal Chemistry

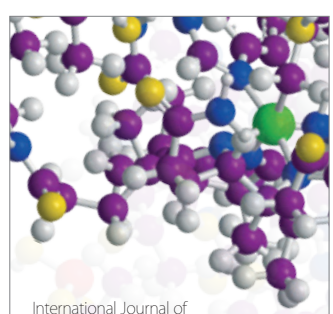

Carbohydrate Chemistry

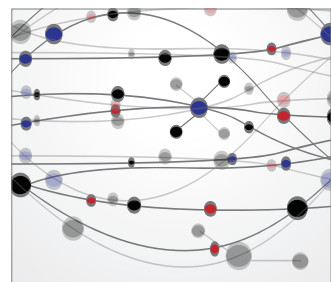

The Scientific World Journal
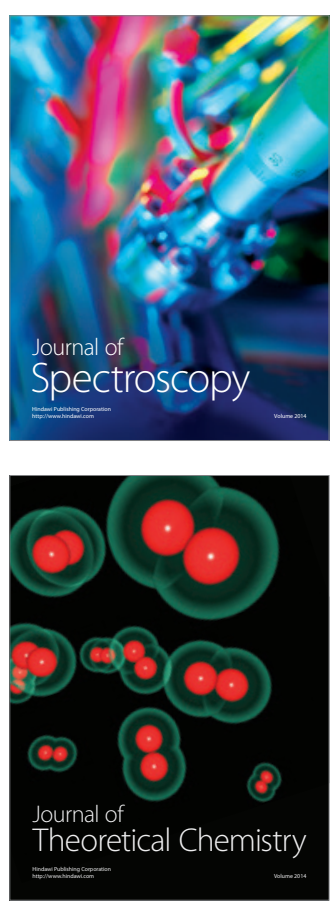
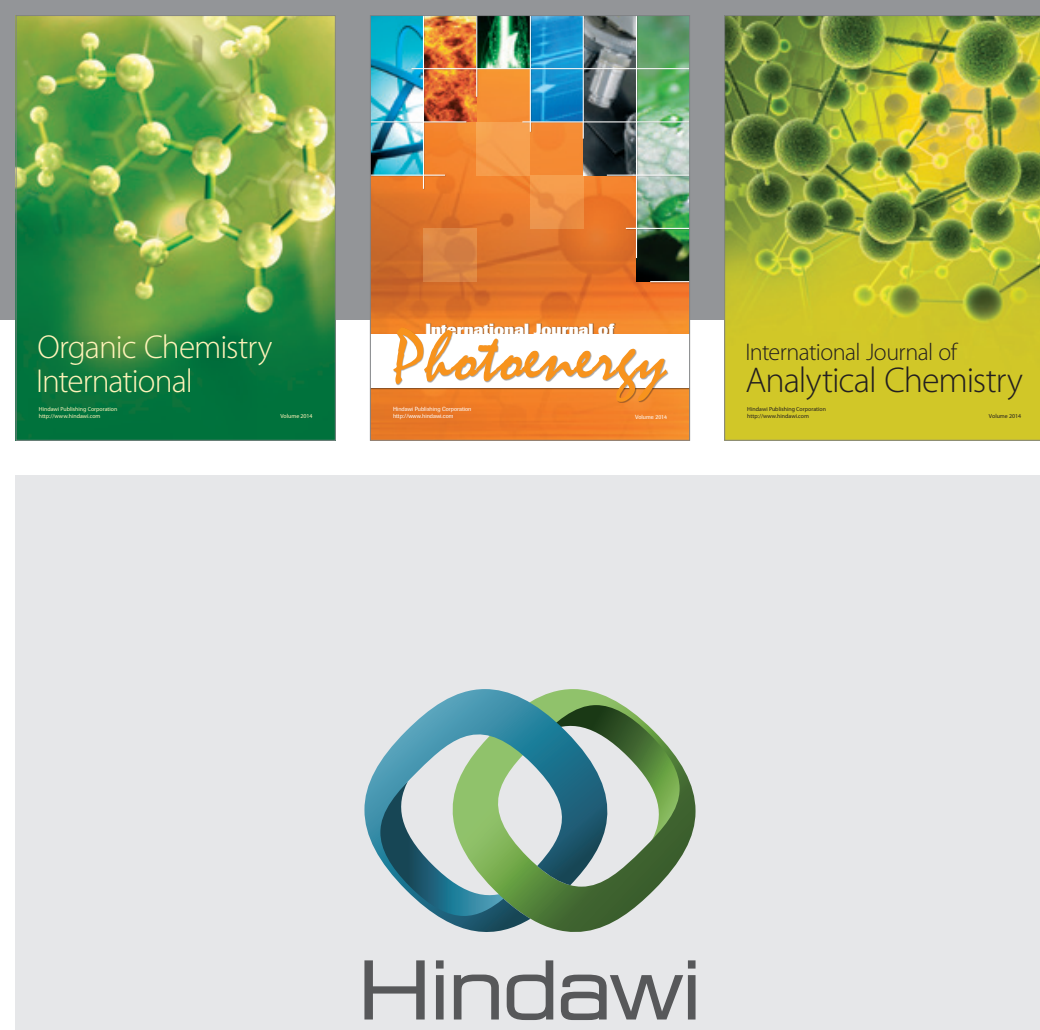

Submit your manuscripts at

http://www.hindawi.com
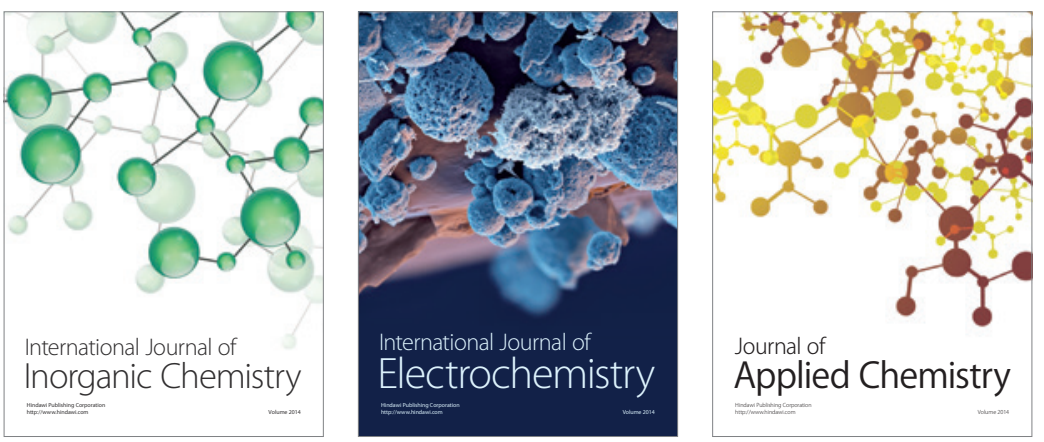

Journal of

Applied Chemistry
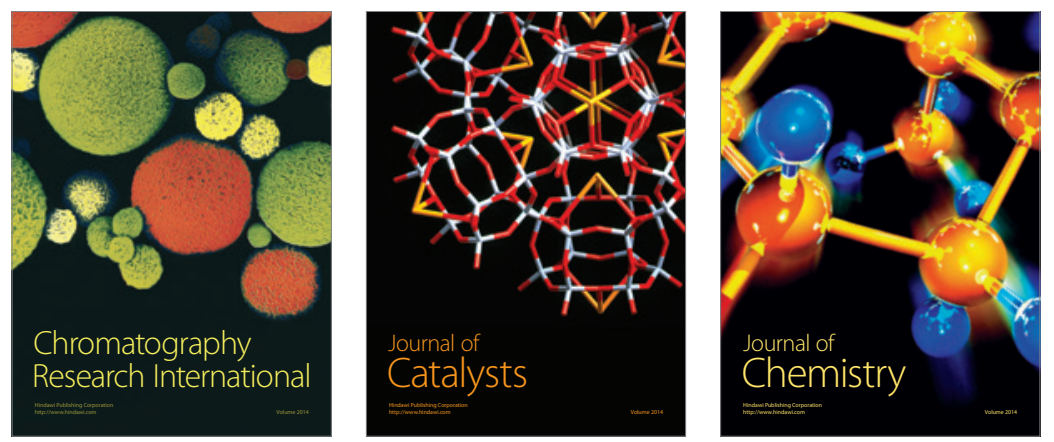
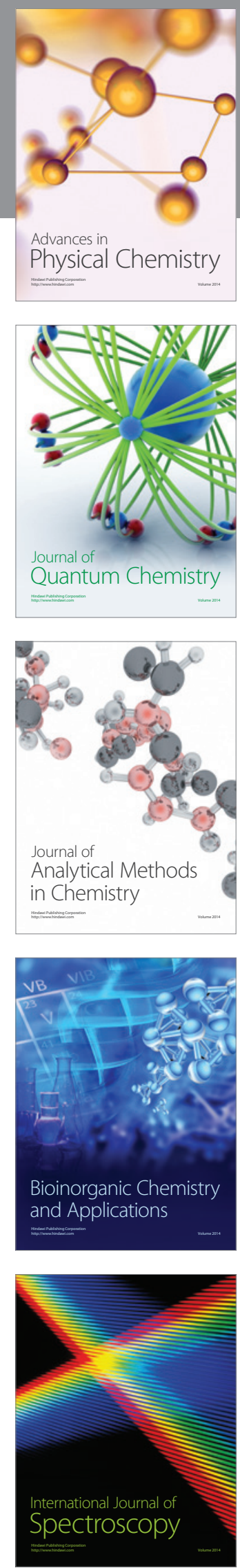